\title{
Posterior reversible encephalopathy syndrome (PRES): A rare disease; review of literature
}

\author{
Nidhi Jain ${ }^{1, *}$, Deepak Goel², Jyotsna Kamra ${ }^{3}$ \\ ${ }^{\mathbf{1}}$ Assistant Professor, ${ }^{\mathbf{3}}$ Associate Professor, Dept. of Obstetrics and Gynecology, Maharaja Agrasen Medical College, Agroha, \\ Hisar, Haryana, ${ }^{2}$ Senior Resident, Dept. of Radiology, Pandit Bhagwat Dayal Sharma Post Graduate Institute of Medical \\ Sciences, Rohtak, Haryana, India
}

*Corresponding Author:

Email: nidhijain270587@gmail.com

Received: $9^{\text {th }}$ March, 2018

Accepted: $8^{\text {th }}$ May, 2018

\begin{abstract}
Posterior reversible encephalopathy syndrome (PRES) is a complex multifactorial disorder diagnosed by clinical symptoms and radiological features. Clinically it presents with headache, diminution of vision, altered mental status, seizures and prolonged comatose state. Diagnosis is confirmed by brain imaging studies (CT/MRI) by presence of vasogenic edema of white matter in parietal and occipital lobes. It is commonly seen in young aged adults with female preponderance. Most commonly it is seen in woman with hypertension and preeclampsia/eclampsia. Early diagnosis and prompt treatment helps in better management of syndrome and thereby preventing the permanent complications. Authors here review the literature via discussing a case of 20 year old pregnant woman who came with ante partum eclampsia and was diagnosed with PRES syndrome.
\end{abstract}

Keywords: Eclampsia, Encephalopathy, Headache, Preeclampsia, PRES.

\section{Introduction}

Posterior reversible encephalopathy syndrome (PRES) is a clinic-radiological syndrome diagnosed by clinical symptoms and radiological features. Clinically patient presents with headache, diminution of vision, altered mental status, seizures and prolonged comatose state. Diagnosis is confirmed on CT/ MRI brain by presence of vasogenic edema of white matter in parietal and occipital lobes.

It was first described by Hinchey et $\mathrm{al}^{1}$ in 1996. Since then few cases have been reported. Now the incidence of PRES has risen due to more vigilance of clinicians and availability of better diagnostic modalities.

It is associated with various clinical conditions including hypertension, preeclampsia, eclampsia, tumour lysis syndrome, blood transfusion, post transplantation and autoimmune diseases. It is most commonly seen in patients with hypertension. It is seen in patients with age ranging from 4-90 years with more preponderance in young aged adults. ${ }^{2}$ It is seen more commonly in females. ${ }^{2}$

Management includes symptomatic treatment including ICU care, control of hypertension, antiepileptic measures and hemodynamic management. Although the syndrome is classically described as reversible entity with active management however permanent complications, mortalities and recurrence of symptoms have been reported. ${ }^{3}$

Hence, early diagnosis and prompt treatment is required for reduction of morbidity and mortality.

\section{Case}

A 20 year old woman, primigravida, was brought to the emergency department of our institution with history of single episode of generalised tonic-clonic seizures and altered mental status since 4 hours. She had history of amenorrhea since 9 months. She was an unbooked case and had no prior history of antenatal visits. There was no prior significant medical history of hypertension and epilepsy in past.

On examination, patient was in post ictal state, drowsy, not obeying verbal commands but responding to painful stimuli. Her pulse was $98 /$ minute and blood pressure was $170 / 110 \mathrm{mmHg}$. Deep tendon reflexes were present and withdrawal response was seen on plantar reflex. On per abdominal examination, single live intrauterine fetus with cephalic presentation was palpated with irregular heart beat of 110 beats/minute.

Primary management including securing wide bore intravenous line, intravenous fluids and intravenous antibiotics, were given. Patient was immediately intubated and was on total ventilator support. Urinary catheterisation was done. Blood investigations were done. After checking deep tendon reflexes, magnesium sulphate was given according to Pritchard's regimen (as shown in Table 1).

Table 1: Clinical conditions associated with PRES

\begin{tabular}{|ll|}
\hline 1. & Hypertension \\
\hline 2. & Preeclampsia/ eclampsia \\
\hline 3. & Autoimmune diseases (SLE, SS, AIDS) \\
\hline 4. & Septicaemia \\
\hline 5. & Post transplantation (renal/ bone marrow) \\
\hline 6. & Acute intermittent porphyria \\
\hline 7. & Renal disease \\
\hline 8. & Cancer chemotherapy \\
\hline 9. & Post blood transfusion \\
\hline
\end{tabular}


Laboratory investigations revealed haemoglobin levels as $8 \mathrm{~g} / \mathrm{dl}$, total leukocyte count $11,000 / \mathrm{dl}$, normal platelet count 1.8 lakhs. Other investigations including liver function test, kidney function test, and coagulation profile were normal. Urine examination revealed proteinuria. Arterial blood analysis was normal.

After taking an informed consent, patient was immediately taken for caesarean section due to fetal distress. A male baby of $1.8 \mathrm{~kg}$ was born with Apgar score 5, 9. Baby was shifted to NICU and mother was shifted to ICU. 1 unit blood and 6 units fresh frozen plasma was given postoperatively. Intravenous injection mannitol was given along with antibiotics, antihypertensive and antiepileptic drugs.

NCCT head was done, which showed ill defined hypo densities in bilateral parietal-occipital region, predominantly on right side, suggestive of PRES as shown in Fig. 1, 2, and 3.

Patient was on ventilator support for two days. On day 3, patient was on oxygen by mask. On day 5, patient was having normal breathing without any oxygen support. Patient was on intravenous antihypertensive (labetolol) for three days after which she was started on oral antihypertensive (tablet telmisartan and hydrochlorthiazide). Patient improved symptomatically in form of normal sensorium, normal vital signs and laboratory investigations. Patient was discharged on day 10 of admission. Patient followed up after 1 week of discharge, which was uneventful.

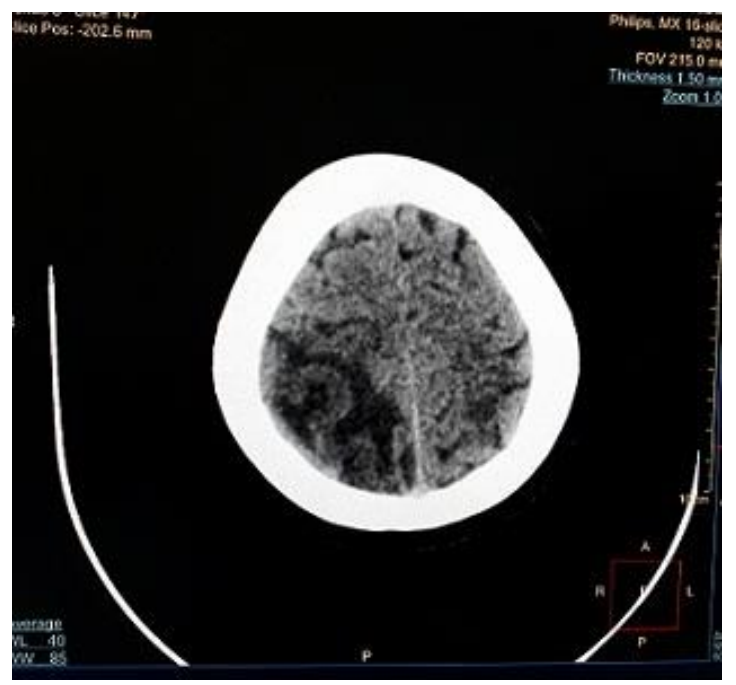

Fig. 1: Axial view of NCCT head showing ill defined hypo densities in right parietal-occipital lobe

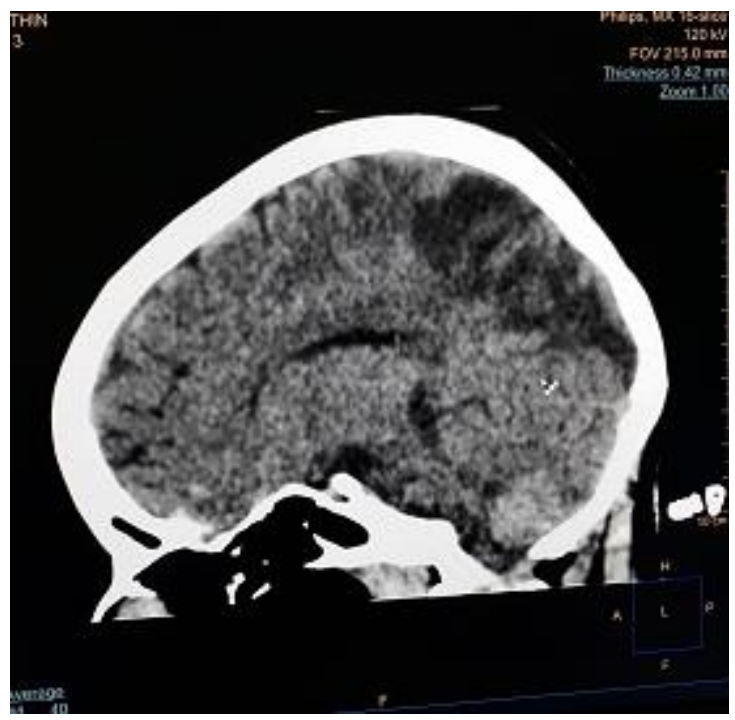

Fig. 2: Sagittal view of NCCT head showing ill defined hypo densities in right parietal-occipital lobe

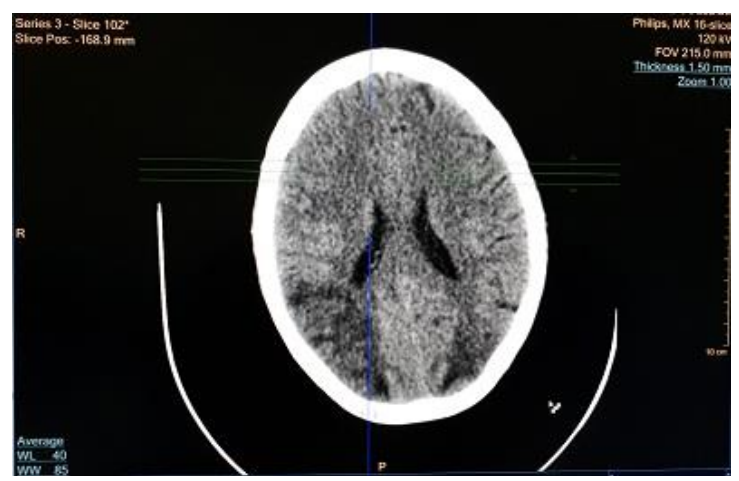

Fig. 3: Axial view of NCCT head showing ill defined hypo densities in bilateral parietal-occipital lobe

\section{Discussion}

PRES is a complex multifactorial reversible syndrome, diagnosed classically by brain imaging studies.

Pathophysiology: The exact pathophysiology of PRES is still unclear however various hypotheses have been given to understand the underlying pathology. Various hypotheses are as follows. ${ }^{4-5}$

1. Failure of cerebral autoregulation with vasogenic edema.

2. Cerebral vasoconstriction leading to brain infarcts.

3. Blood brain barrier disruption due to endothelial damage leading to fluid and protein denudation.

In 2012, in a study by Esther V. Hobson ${ }^{6}$ et al, the most popular theory of PRES syndrome has been described as uncontrolled severe hyerpertension leading to interruption to brain auto regulation. The authors have described that cerebral blood flow or perfusion in auto regulated by dilatation and constriction of vessels. This auto regulation is disrupted when mean arterial pressure (MAP) is more than $150-160 \mathrm{mmHg}$. In patients with chronic hypertension, this threshold value of MAP is comparatively higher. Hence, due to 
uncontrolled hypertension, breakdown of auto regulation occurs, which leads to hyper perfusion, resulting in extravasation of proteins and fluid in interstitial area and thereby, causing cerebral vasogenic edema. This stage is still reversible. But when MAP is more than $200 \mathrm{mmHg}$, it becomes irreversible.

In an article by W.S. Bartynski, ${ }^{7}$ theories of pathophysiology have been compared. Observations which were supporting the theory of hypertension/ hyper perfusion were that PRES syndrome is primarily related with presence of significant elevated blood pressure. In $50-70 \%$ patients, moderate to severe hypertension is found. Also, management of hypertension leads to symptomatic improvement of patient within weeks. But point which disfavour the theory is, in $20-30 \%$ of patients with PREs syndrome are found to be normotensive. Another theory was systemic toxicity leading to endothelial dysfunction with subsequent vasoconstriction or leukocyte trafficking or both. Point which favours this theory is that PRES syndrome is usually associated with sepsis, transplantation and autoimmune diseases.

So, various theories are available to explain mechanism in different patients. However, one theory which can explain the pathophysiology of PRES syndrome in all patients is still unavailable.

Associated Conditions: Various clinical conditions are associated with PRES syndrome as listed in table 1. However, most commonly it is seen in women with hypertensive disorders, preeclampsia and eclampsia.

Few cases have been reported in patients with blood transfusion. ${ }^{8-10}$ The underlying pathology is explained as rapid increase in total blood flow due to blood transfusion, leading to cerebral overload. In our patient, there was history of ante partum eclampsia and blood transfusion was given post operatively, which can be the contributory factor to PRES.

A case was reported by Bavikatte $\mathrm{G}$ et $\mathrm{al}^{11}$ in 2010 , in which PRES syndrome was found as a complication of Gullain-Barre syndrome. A 62 year old woman presented with sudden onset of complete bilateral visual loss with hypertension (BP: 204/113 mm Hg) and flaccid weakness of all four limbs. Her CSF examination and nerve conduction studies were done. She was diagnosed as case of Gullain-Barre syndrome (GBS). On MRI Brain, she was found to have classic features of PRES syndrome.

Diagnosis: Diagnosis of PRES is based on presence of classical clinical symptoms and findings on CT/MRI brain.

In emergency cases, CT scan helps in rapid evaluation of patient. It also helps in excluding any cerebral haemorrhage or space occupying lesion. However, it is not $100 \%$ sensitive to diagnose PRES syndrome.

MRI is the imaging modality of choice. ${ }^{12}$ It is characterised by presence of high signal intensity in white matter of posterior regions of both cerebral hemispheres. It is seen predominantly in parietal and occipital lobes. In our patient, NCCT head was done which showed hypo densities in bilateral parietaloccipital lobes.

However, some atypical features such as haemorrhage, cortical lesions or isolated frontal lobe involvement have also been described on MRI.

In study by McKinney at al, ${ }^{13}$ atypical MRI findings of PRES syndrome has been described.76 patients with PRES syndrome were evaluated on MRI. It was found that most common region involved was parietooccipital $(98.7 \%)$ followed by posterior frontal, temporal, thalamus, cerebellum, brainstem and basal ganglia. Atypical manifestations were found as enhancement, restricted diffusion and haemorrhage.

Management: The management of PRES includes early diagnosis, symptomatic management and correction of underlying clinical condition. Since maximum numbers of cases are found to be associated with hypertension, hence aggressive control of blood pressure helps in rapid reversal of changes. Ventilator support is required to protect the airways. Antiepileptic drugs are given to prevent occurrence or to treat seizures. If managed promptly and appropriately, disease is reversible in majority of cases.

Prognosis: PRES syndrome carries an excellent prognosis if diagnosed and managed promptly. The clinical features usually resolves within a week and radiological imaging features might take three to four weeks to resolve. However, rarely prolonged neurological deficit and recurrent episodes has been reported.

\section{Conclusion}

PRES is a reversible syndrome which requires high degree of suspicion for early diagnosis. So, in a case with headache, seizures, visual disturbances or neurological deficit, a clinician must be vigilant regarding PRES syndrome. Brain imaging study is crucial for its diagnosis. If diagnosed at an early reversible stage and managed timely, the underlying pathology can be rectified and thereby, preventing the irreversible neurological sequel and fatalities. Hence, early diagnosis and prompt treatment is the key to manage a case of PRES.

\section{Conflict of Interest: none.}

\section{Abbreviations}

PRES: Posterior reversible encephalopathy syndrome, CT: computed tomography

MRI: magnetic resonance imaging

ICU: intensive care unit

SLE: systemic lupus erythematous

SS: systemic sclerosis

AIDS: acquired immunodeficiency syndrome. 


\section{References}

1. Rijal JP, Giri S, Dawadi S, Dahal KV. Posterior reversible encephalopathy syndrome (PRES) in a patient with late postpartum eclampsia. BMJ Case Rep. 2014.

2. Legriel S, Pico F, Azoulay E. Understanding posterior reversible encephalopathy syndrome. Annual update in intensive care and emergency medicine 2011. Springer, 2011:631-53.

3. Roth C, Ferbert A. Posterior reversible encephalopathy syndrome: long-term follow-up. J Neurol Neurosurg Psychiatry. 2010;81:773-7.

4. Legriel S, Pico F, Azoulay E. Understanding posterior reversible encephalopathy syndrome. Annual Update in Intensive Care and Emergency Medicine. 2011, 631-653.

5. Bartynski WS. Posterior reversible encephalopathy syndrome, part 1: fundamental imaging and clinical features. AJNR Am J Neuroradiol. 2008;29(6):1036-1042.

6. Hobson EV, Craven I, Blank SC. Posterior reversible encephalopathy syndrome: a truly treatable neurologic illness. Perit Dial Int. 2012;32(6):590-594.

7. W.S. Bartynski. Posterior reversible encephalopathy syndrome, part 2: controversies surrounding pathophysiology of vasogenic edema. AJNR Am J Neuroradiol. 2008;29(6):1043-1049

8. Huang YC, Tsai PL, Yeh JH, Chen WH. Reversible posterior leukoencephalopathy syndrome caused by blood transfusion: a case report. Acta Neurol Taiwan. 2008;17(4):258-262.
9. Sato Y, Hirose M, Inoue Y, Komukai D, Takayasu M,Kawashima E, Koiwa F, et al. Reversible posterior leukoencephalopathy syndrome after blood transfusion in a patient with end-stage renal disease. Clin Exp Nephrol. 2011;15(6):942-947.

10. Wada K, Kano M, Machida Y, Hattori N, Miwa H. Posterior reversible encephalopathy syndrome induced after blood transfusion for severe anemia. Case Reports in Clinical Medicine. 2013;2:332-334.

11. Bavikatte G, Gaber T, Eshiett MU. Posterior reversible encephalopathy syndrome as a complication of GullainBarre syndromr. J Clin Neurosci. 2010; 17:924-6.

12. Pedraza R., Marik P. E., Varon J. Posterior reversible encephalopathy syndrome: a review. Critical Care \& Shock. 2009;12(4):135-143.

13. McKinney AM, Short J, Truwit CL, McKinney ZJ, Kozak OS, SantaCruz KS et al. Posterior reversible encephalopathy syndrome: incidence of atypical regions of involvement and imaging findings. AJR Am J Roentgenol. 2007;189:904-12.

How to cite this article: Jain $\mathrm{N}$, Goel $\mathrm{D}$, Kamra J. Posterior reversible encephalopathy syndrome (PRES): A rare disease; review of literature. Ind $\mathbf{J}$ Obstet Gynecol Res. 2018;5(3):314-317. 